\title{
One-Way Light Speed Determination Using the Range Measurement Equation of the GPS
}

\author{
Stephan J. G. Gift \\ Department of Electrical and Computer Engineering \\ Faculty of Engineering \\ The University of the West Indies \\ St. Augustine, Trinidad and Tobago, West Indies \\ Tel: 868-662-2002 ext 2166 E-mail: Stephan.Gift@sta.uwi.edu
}

Received: November 29, 2010 Accepted: December 17, 2010 doi:10.5539/apr.v3n1p110

\begin{abstract}
The one-way speed of light is determined using the range measurement equation of the Global Positioning System. This equation has been rigorously and extensively tested and verified in the Earth-Centred Inertial frame, a frame that moves with the Earth as it revolves around the Sun but does not share its rotation. The result is a simple demonstration of one-way light speed anisotropy depending on the direction of propagation relative to the rotating Earth.
\end{abstract}

Keywords: Special relativity, One-way, Light speed, Constancy, Anisotropy, GPS, Range measurement, Equation, Synchronization

\section{Introduction}

The constancy of the speed of light in all inertial frames is a fundamental postulate of Einstein's Special Theory of Relativity and a major principle of modern physics [French, A.P., 1968; Rindler, W., 1991; Williams, 2002]. While the principle is defined in inertial frames, its application in the non-inertial frame of the Earth's surface such as in the Einstein clock-synchronization procedure and the SI unit of length is accepted science since it would be virtually useless otherwise. Indeed the overwhelming majority of experiments testing the postulate including have been performed in the non-inertial terrestrial frame. These include the modern Michelson-Morley type experiments such as those by Brillet and Hall [Brillet, A., 1979], Muller [2003], Wolfe [2004], Hermann [2005], Antonini et al. [2005], Eisele et al. [2009], Hermann et al. [2009]. However while light speed invariance has been experimentally established for two-way light transmission, the one-way speed of light has not been properly tested since this requires clock synchronization that it has not been possible to achieve [Zhang, 1997; Gezari, 2010]. Experiments such as those by Gagnon et.al. [1988], Krisher et.al. [1990] and Riis et.al. [1988] have been shown to be invalid one-way light speed tests [Zhang, 1997]. The advent of the Global Positioning System (GPS) has changed the situation.

The GPS is a modern timing-ranging system with accurate synchronized atomic clocks that enable precise determination of position on the Earth. Wolf and Petit [1997] used this system to test the isotropy of the speed of light and published a limit of $\delta c / c<5 \times 10^{-9}$. This result however holds for the Earth-Centered Inertial frame or ECI frame in which the test was conducted and does not necessarily apply to other inertial frames moving relative to the ECI frame where the light speed may vary. Based on GPS timing, Marmet [2000] observed that a light signal takes about 14 nanoseconds longer than the average time (where average time equals distance divided by $c$ ) traveling Eastward from San Francisco to New York while the signal takes about 14 nanoseconds less than the average time traveling Westward from New York to San Francisco. Kelly [2005] also noted that time measurements using the GPS show that a light signal takes 207.4 nanoseconds longer to circumnavigate the Earth Eastward at the equator than the average time while a light signal takes 207.4 nanoseconds less in the Westward direction around the same path.

Both researchers concluded that these observed travel time differences in each direction arise because light travels at speed $c-v$ Eastward and $c+v$ Westward relative to the surface of the earth where $v$ is the Earth's rotational speed at the particular latitude. They however did not publish any calculations perhaps because of the 
problems presented by measurement errors. Using the synchronized clocks of the GPS in a novel application, Gift [2010] overcame these problems and directly confirmed light speed anisotropy $c \pm v$ in the East-West direction. In this paper we again test light speed constancy using a different feature of the GPS. Specifically, we utilise the range measurement equation of the GPS to examine light speed isotropy between two adjacent points fixed at the same latitude on the surface of the rotating Earth.

\section{Light Speed Determination using the Range Measurement Equation}

The range measurement equation is central to the operation of the GPS. It holds in an ECI frame which is a frame that moves with the Earth as it revolves around the Sun but does not share its rotation. It is given by [Ashby, 2003]

$$
\left|\overline{r_{r}}\left(t_{r}\right)-\overline{r_{s}}\left(t_{s}\right)\right|=c\left(t_{r}-t_{s}\right)
$$

where $t_{s}$ is the time of transmission of an electromagnetic signal from a source, $t_{r}$ is the time of reception of the electromagnetic signal by a receiver, $\bar{r}_{s}\left(t_{s}\right)$ is the position of the source at the time of transmission of the signal and $\overline{r_{r}}\left(t_{r}\right)$ is the position of the receiver at the time of reception of the signal. Using elapsed time measurements determined by the GPS clocks and the light speed value $c$, this equation enables the accurate determination of position on the surface of the Earth. It has been extensively and rigorously tested and verified. The appearance of $c$ in the equation is sometimes interpreted as a demonstration of the principle of light speed constancy in an inertial frame [Ashby, 1994]. However Wang [2000] used the range measurement equation operating in an ECI frame to show that the speed of light is dependent on the observer's uniform motion relative to the ECI frame. He did this by using the range measurement equation to determine elapsed time and concluded that the successful application of the range measurement equation in GPS operation is inconsistent with the principle of the constancy of the speed of light.

Following Wang's approach we use the range measurement equation to determine elapsed time for light traveling between two adjacent points fixed on the surface of the rotating Earth at the same latitude and use this time and the known distance between the two fixed points to determine the one-way speed of light. Thus, consider two adjacent GPS stations A and B at the same latitude and fixed on the surface of the Earth a distance $D$ apart with B East of A. Since the Earth is rotating, the stations are moving eastward at speed $v$ the Earth's surface speed at that latitude. Let $D$ be sufficiently small (stations A and B in the same building) such that the stations are moving uniformly in the same direction at speed $v$ relative to the ECI frame. In such circumstances stations A and B constitute an (approximately) inertial frame moving at speed $v$ relative to the ECI frame.

\subsection{Eastward Transmission}

Let station A transmit a signal eastward at time $t_{I}$ to station B which receives it at time $t_{F}$. On an axis fixed in the ECI frame along the line joining the two stations with the origin west of station $\mathrm{A}$, let $x_{A}(t)$ be the position of station $\mathrm{A}$ at time $t$ and $x_{B}(t)$ be the position of station $\mathrm{B}$ at time $t$. Then from the range measurement equation (1),

$$
x_{B}\left(t_{F}\right)-x_{A}\left(t_{I}\right)=c\left(t_{F}-t_{I}\right)
$$

where $x_{B}\left(t_{F}\right)$ is the position of station B at time $t_{F}$ and $x_{A}\left(t_{I}\right)$ is the position of station A at time $t_{I}$. Since the stations are moving uniformly in the same direction at speed $v$ relative to the ECI frame, it follows that the relation between the position $x_{B}\left(t_{F}\right)$ of station $\mathrm{B}$ at the time of reception of the signal and its position $x_{B}\left(t_{I}\right)$ at the time of emission of the signal is given by

$$
x_{B}\left(t_{F}\right)=x_{B}\left(t_{I}\right)+v\left(t_{F}-t_{I}\right)
$$

Substituting for $x_{B}\left(t_{F}\right)$ from (3) in (2) yields

$$
x_{B}\left(t_{I}\right)-x_{A}\left(t_{I}\right)+v\left(t_{F}-t_{I}\right)=c\left(t_{F}-t_{I}\right)
$$

This becomes 


$$
x_{B}\left(t_{I}\right)-x_{A}\left(t_{I}\right)=D=(c-v)\left(t_{F}-t_{I}\right)
$$

giving the elapsed time as

$$
\left(t_{F}-t_{I}\right)=\frac{D}{c-v}
$$

Therefore the speed $c_{A B}$ of the light traveling from station A to station B is given by separation $D$ divided by elapsed time $\left(t_{F}-t_{I}\right)$ which using $(6)$ is

$$
c_{A B}=\frac{D}{\left(t_{F}-t_{I}\right)}=\frac{D}{D /(c-v)}=c-v
$$

\subsection{Westward Transmission}

Let station B transmit a signal westward at time $t_{I}$ to station A which receives it at time $t_{F}$. Then using the range measurement equation (1) and noting that $x_{B}\left(t_{I}\right)>x_{A}\left(t_{F}\right)$,

$$
x_{B}\left(t_{I}\right)-x_{A}\left(t_{F}\right)=c\left(t_{F}-t_{I}\right)
$$

where $x_{B}\left(t_{I}\right)$ is the position of station B at time $t_{I}$ and $x_{A}\left(t_{F}\right)$ is the position of station A at time $t_{F}$. Since the stations are moving uniformly in the same direction at speed $v$ relative to the ECI frame, the relation between the position $x_{A}\left(t_{F}\right)$ of station $\mathrm{A}$ at the time of reception of the signal and its position $x_{A}\left(t_{I}\right)$ at the time of emission of the signal is given by

$$
x_{A}\left(t_{F}\right)=x_{A}\left(t_{I}\right)+v\left(t_{F}-t_{I}\right)
$$

Substituting for $x_{A}\left(t_{F}\right)$ from (9) in (8) yields

$$
x_{B}\left(t_{I}\right)-x_{A}\left(t_{I}\right)-v\left(t_{F}-t_{I}\right)=c\left(t_{F}-t_{I}\right)
$$

This becomes

$$
x_{B}\left(t_{I}\right)-x_{A}\left(t_{I}\right)=D=(c+v)\left(t_{F}-t_{I}\right)
$$

giving the elapsed time as

$$
\left(t_{F}-t_{I}\right)=\frac{D}{c+v}
$$

Therefore the speed $c_{B A}$ of the light traveling from station B to station A is given by separation D divided by elapsed time $\left(t_{F}-t_{I}\right)$ which using (12) is

$$
c_{B A}=\frac{D}{\left(t_{F}-t_{I}\right)}=\frac{D}{D /(c+v)}=c+v
$$

\section{Discussion}

The results in equations (7) and (13) indicate that light travels faster westward than eastward relative to the surface of the Earth. In particular the one-way determination of light speed using the range measurement equation of the GPS establishes in (7) that a signal sent eastward travels at speed $c$ minus the rotational speed of the Earth $v$ at that latitude giving $c-v$. The GPS data also shows in (13) that a signal sent westward travels at speed $c$ plus the rotational speed of the Earth $v$ at that latitude giving $c+v$. This is true for the short-distance inertial frame considered in this paper as well as long-distance circumnavigation of the Earth [Kelly, 2005]. Similar light speed anisotropy $c \pm w$ was observed in the Roemer [Gift, 2010] and Doppler [Gift, 2010] experiments involving the revolution of the Earth at speed $w$ in its approximately linear motion around the Sun. 
The one-way light speeds $c \pm v$ determined using the GPS range measurement equation were not detected by the many light speed experiments conducted in the terrestrial frame which all give light speed $c$ but are exactly the results obtained using the GPS synchronized clocks [Gift, 2010]. These changed light speed values $c \pm v$ contradict the principle of the constancy of the speed of light applied in the terrestrial frame where the principle requires constant light speed $c$ for light traveling westward or eastward between the two adjacent stations. The timing differences arising from the varying light speed on the surface of the Earth are actually programmed in the GPS computers in order to produce accurate global positioning.

\section{Conclusion}

In this paper, the range measurement equation of the GPS was used to determine the one-way light speed between two adjacent points at the same latitude and fixed on the surface of the Earth. The result $c \pm v$ represents an unmistakable variation in the speed of light arising from the rotation of the Earth as the light travels in the east-west direction and is consistent with light speed anisotropy results previously obtained [Marmet, 2000; Kelly, 2005; Gift, 2010]. It is a direct repudiation of the application of the principle of light speed constancy in the non-inertial frame of the surface of the Earth which is today done routinely. This confirms the light speed variation claim made previously by this author in [Gift, 2006] and demolishes the strident criticisms of that claim by Osborne [2006], Klauber [2006] and Flanagan and Terzian [2006].

\section{References}

Antonini, P., M. Okhapkin, E. Goklu and S. Schiller. (2005). Test of Constancy of Speed of Light With Rotating Cryogenic Optical Resonators, Physical Review A, 71, 050101.

Ashby, N. (1994). Relativity in the Future of Engineering, IEEE Trans. on Instrumentation and Measurement, 43, 505 .

Ashby, N. (2003). Relativity in the Global Positioning System, Living Reviews in Relativity, 6, 1.

Brillet, A. and Hall, J.L., Improved Laser Test of the Isotropy of Space, Phys. Rev. Lett., 42, 549, 1979.

Eisele, C, Nevsky, A. and Schiller, S. (2009). Laboratory Test of the Isotropy of Light Propagation at the $10^{-17}$ Level, Physical Review Letters, 103, 090401.

Flanagan, E. and Terzian, Y. (2006). Gift is in Error, Journal of Scientific Exploration, 20, 608.

French, A.P. (1968). Special Relativity, Nelson, London.

Gagnon, D.R., Torr, D.G., Kolen, P.T. and Chang, T. (1988). Guided-Wave Measurement of the one-way Speed of Light, Physical Review A, 38, 1767.

Gezari, D. Y. (2010). Experimental Basis for Special Relativity in the Photon Sector, arXiv: 0912.3818.

Gift, S.J.G. (2006). The Relative Motion of the Earth and the Ether Detected, Journal of Scientific Exploration, $20,201$.

Gift, S.J.G. (2010). Doppler Shift Reveals Light Speed Variation, Apeiron 17, 13.

Gift, S.J.G. (2010). Light Speed Invariance is a Remarkable Illusion, Physics Essays, 23, 1.

Gift, S.J.G. (2010). One-Way Light Speed Measurement Using the Synchronized Clocks of the Global Positioning System (GPS), Physics Essays, Vol.23, No2, pp 271-275, June.

Hermann, S., A. Senger, E. Kovalchuk, H. Muller and A. Peters. (2005). Test of the Isotropy of the Speed of Light Using a Continuously Rotating Optical Resonator, Physical Review Letters, 95, 150401.

Herrmann, S. et al. (2009). Rotating optical cavity experiment testing Lorentz invariance at the $10^{-17}$ level, Physical Review, D 80, 105011.

Kelly, A. (2005). Challenging Modern Physics, BrownWalker Press, Florida.

Klauber, R. (2006). Detection of the Ether?, Journal of Scientific Exploration, 20, 601.

Krisher, T.P., Maleki, L., Lutes, G.F., Primas, L.E., Logan, R.T., Anderson, J.D. and Will, C.M. (1990). Test of the Isotropy of the One-way Speed of Light Using Hydrogen-Maser Frequency Standards, Phys. Rev, D 42, 731.

M"uller, H., Herrmann, S., Braxmaier, C and Peters, A. (2003). Modern Michelson-Morley Experiment using Cryogenic Optical Resonators, Phys. Rev. Lett., 91, 020401.

Marmet, P. (2000). The GPS and the Constant Velocity of Light, Acta Scientiarum, 22, 1269. 
Osborne, M. (2006). Response to "The Relative Motion of the Earth and the Ether Detected" by Stephan J.G. Gift, Journal of Scientific Exploration, 20, 597.

Riis, E., Lars-Ulrik, A.A., Bjerre, N and Poulsen, O. (1988). Test of the Speed of Light Using Fast-Beam Laser Spectroscopy, Physical Review Letters, 60, 81.

Rindler, W. (1991). Introduction to Special Relativity, Clarendon Press, Oxford.

Wang, R. (2000). Successful GPS Operations Contradict the Two Principles of Special Relativity and Imply a New Way for Inertial Navigation-Measuring Speed Directly, Proceedings of the IAN World Congress in Association with the U.S. ION Annual Meeting, 26-28 June, San Diego, CA.

Williams, W.S.C. (2002). Introducing Special Relativity, Taylor and Francis, London.

Wolf, P. and Petit, G. (1997). Satellite Test of Special Relativity Using the Global Positioning System, Physical Review A, 56, 4405.

Wolf, P. et al. (2004). Improved Test of Lorentz Invariance in Electrodynamics Physical Review D, 70, 051902.

Zhang, Y.Z. (1997). Special Relativity and its Experimental Foundations, World Scientific, Singapore. 